\title{
Subject Index, Vol. 34, 1972
}

Acid monoamine metabolites 136

Acromegaly 412

Adaptation 224

Amygdala 176

Aneurysm thrombosis 368

Athetosis 34

Athetosis, clinical picture 7

Behavior 210

Behaviour disturbances 176

Bereitschaftspotential 224

Bradykinesia 130

Brain atrophy 285

Brain displacements 348

Brain impedance 112

Brain oxygen tension 112

Brain tumour: localisation, biopsy, intra-

tumoural electrical activity 101 Brain polarography 112 Brain positional changes 348

Brain probing 112

Caudate nuclei 237 Centre median nucleus 265 Centrencephalic epilepsy 252

Cephalicpajn 382 Cerebral dominance 162 Cerebral palsy 18 Cerebral palsy, clinical picture 7 Cerebrospinal fluid 136 Cervical cord 299 
Choreoathetosis/Huntington's chorea 74

Cingulum 398

Closed circuit 218

CNV (contingent negative variation) 224

Cognitive regulation 162

Color word test 162

Combined thalamo-subthalamotomy 55

Comparison of postoperative results with

long-term results 41 Computer 94 Conray 34

Corpus callosum 169, 237 Corticospinal tract 230 Criteria of death 112 Cryosurgery 74

Depth EEG 101

Depth electrode 252

Depth electrography 363

Diabetes mellitus 355

Differentiated electrophysiological stimula-

tion 55 DifFuse slowing of EEG 51 Dihydromorphanthridine derivative 130 Direct PO2

measurement in brain tissue, in

subcortical structures, in brain tumors,

parkinsonism, hyperkinesias 106 L-Dopa 55,130 L-Dopa in Parkinsonism 143

Dopamine metabolism 281

Doublé athetosis 18 
Subject Index 
Drug administration 295 Dystonia musculorum deformans 34

Electrical stimulation 197, 230 ElectroencephalQgram 64, 112, 525

Electroencephalography 14 Electromyography 230, 295 Epilepsy 169, 200 Epileptiform potentials 64 Evoked potentials 389 Evoked potential of EEG 84 Extrapyramidal disorders 200 Extrapyramidal hyperkinesia 325 Extrapyramidal- myoclonias 57

Fluorescence microscopy 143 Functionally developed motor system 51 Functionally undeveloped motor system 51

High frequency stimulation of thalamus andpallidum 51 Hippocampus 398 Histology 210 Homovanillic acid 136 Horner's syndrome, central and peripheral 156 H-reflex 84, 295 Human thalamus 184 5-Hydroxyindoleacetic acid 136 Hydrocortisone 149 Hyperkinesia 29

Hyperkinesias of cerebellar origin 74 Hypertonic dyskinesias 74 Hypokinetic syndromes 55 Hypophysectomy 412 Hypothalamic stimulation 374

Inductive heating in extrapyramidal motor disturbances 325 Interstitial nucleus of Cajal 331 Intracerebral stimulation 64 Intracranial aneurysms 368 Intracranial pressure 136 Intractable pain 264 Intrathalamic application 149 Intraventricular application 149 In vivo study 112 
Involuntary movement disorders 74

Localized cooling 218 Long-term results depending on zona in-certalesions 41

Malignant disease 264

Medial lemniscus 184

Medial longitudinal fasciculus 331

Memory mechanism 398

Meningoencephalitis 339

Microsurgery of lumbar enlargement 311

Midbrain tractotomy 382

Miosis 156

Monkey 374

Motor responses 230

Motor thalamus 224

Myelotomy 299

Myoclonic disease 320

Myoclonic disease of Ramsey-Hunt 315

Myoclonus 64, 74

N. medialis dorsalis 265 Neurogenic bladder 311 New apparatus for spinal cord stereotaxis

311 New transtentorial approach from one side

320 Nucleus dentatus 29

Ocular pressure 156 Operation technique 127 Ophthalmoplegia 355 Oral pain 382

Pain 265

Pain alleviation 374

Pallidal lesions 41

Pallidotomy 152

Parkinsonism 34, 285

Parkinson's disease $55^{\wedge} 136,162,210,281$

Parkinson syndrome 295

Parkinsonian type hyperkineses 14

Pathogenesis of brain disease 14

Pathophysiology 57

Percutaneous 275, 389

Peripheral nerve stimulation 64

Plasma renin 281 
Subject Index

Plethysmography 152 Postoperative physiotherapy 7 Probe 218

Psychological testing 162 Psycho-Orgahic syndrome, postencepha-

litic. 285 Ptosis 156

Pupillary reaction 84 Pyramidotomy 299

Radiofrequency 412

Radiosurgical thalamotomy 264

Raynaud's disease 152

Related subthalamic structures 200

Renin angiotensin system 281

Results of stereotaxic thalamotomy 57

Reversible blockage 218

Rhythmic slow waves of EEG 51

Rigidity 130

Risk patients 285

Row of ventral thalamic nuclei 18

Sagittal thalamotomy 18 See-saw nystagmus 331 Side-protruding electrode 94 Skin resistance, electrical 156 Somatotopic arrangement 169 Somatotopic array 197

Spasmodic torticollis 41, 74 Spasticity 29, 320 Spasticity of the lower limbs 311 Spinal cord pain pathways 275 Spinoquintothalamic tract 184 Spinothalamic tra6ft 275

Spinothalamic tractotomy 299 Stereoencephalotomy 169, 224 Stereotactic 389

Stereotactic dentatotomy 320 Stereotacfcinstruments 299 Stereotactic operation 55, 70, 149,106 Stereotactic operations in extrapyxamid

motor disorders 325 Stereotactic procedures 197, 348 Stereotactic surgery 34,184

Stereotactic thrombosis 368 Stereotactic treatment 41 
fótgjeotaxic amygdalotomy 176

Stereotaxic coagulation 295

Stereotaxic frame 127

Stereotaxic instrumëntation, new 127

Stereotaxic pneumoencephalography 285

ötgreotaxic procedures 295

Stereotaxic semiology 14

Stereotaxic surgery 29, 84, 210

Stereotaxic thalamotomy 265

Stereotaxis 299

Stereotaxy 94, 230

Stimulation 184

Stimulation of thalamus 84

Striatal projection 237

Subcallosal fasciculi 237

Subcortical mechanisms 252

Subthalamic region 252

Subthalamotomy 156

Subthalamus 29, 70, 200

Target 264

Target point 84

Target reliability 84

Temporal lobe epilepsy 176, 363

Telemetry 363

Thalamus 29, 70,162,197, 210

Thermal lesions 412

Torticollis 331

Tractotomy 389

Transitory disappearance of signs 315

Transtentorial bilateral dentatotomy from

theright side 315 Tremor treatment 70, 84, 130 Trigeminal 389 Trigeminal tractotomy 299 L-Tryptophan in Parkinsonism 143

Vacuüm 218 Vascular thrombosis 339 Vegetative central system 281 Ventral oral nucleus lesions 41 Ventriculojgaphy 34 Ventrolateral thalamotomy 162 Ventro-oral nucleus 197 Verbal cognition 162 